\section{A New Balloon System for Complete Dilation of Self-Expanding Mesh Stents in Malignant Esophageal Stenosis}

In the palliative treatment of malignant esophageal stenoses, the implantation of self-expanding wire mesh prostheses has become a recognized therapeutic procedure, and various types of prostheses using different materials are available. The self-expanding nitinol Ultraflex prosthesis, with a diameter of $18 \mathrm{~mm}$ and a length of $70-140 \mathrm{~mm}$ (Microvasive, Boston Scientific) is a well-known example $(1,2)$. However, our own experience with more than 20 patients who exclusively received this type of prosthesis has shown that, in contrast to other types of prostheses, the Ultraflex tends to lead to the formation of folds, due to its slightly lower initial expansion force (particularly if more strength is required and more extensive tumor stenoses are present). These folds occur particularly at the tulip-shaped proximal end of the prosthesis, appearing radiographically as apparent contortions of the mesh. If the prosthesis does not fully unfold, it cannot completely fulfil its function, and dysphagia may persist; in addition, dislocations due to inadequate fixation, particularly at the proximal end, are possible. An additional balloon expansion of the implanted stent is therefore recommended in theses cases. Due to their potential for eccentric expansion and shortening during air insufflation, however, the balloon catheter systems currently available have been associated with a risk of stent dislocation. A new balloon catheter system with a concentrically expanding balloon consisting of nylon-reinforced polyurethane has therefore proved valuable in our experience. The newly developed Olbert Catheter System for esophageal dilation (Meadox-Surgimed, Denmark) is a coaxial catheter (total length: $120 \mathrm{~cm}$, shaft: $10 \mathrm{Fr}$ Teflon tube) with an expandable balloon at the distal end (Figure 1). The inner lumen accepts a 0.038 -inch guidewire. The diameter of the unexpanded balloon is approximately $22 \mathrm{Fr}(7.3 \mathrm{~mm})$ and is $8 \mathrm{~cm}$ in length, with an inflated diameter of $20.5 \mathrm{~mm}(60 \mathrm{Fr})$ at the maximum inflation pressure of six atmospheres (ca. $600 \mathrm{kPa}$ ). The distal and proximal ends of the balloon are delineated by two radiopaque gold markers.

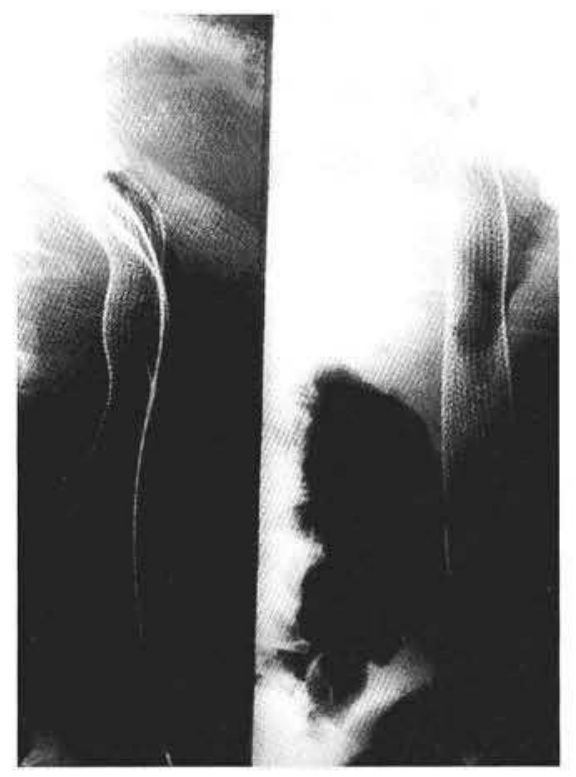

Figure 1: An implanted Ultraflex pros thesis with incomplete unfolding at the proximal end in an extensive stenosis. Left: before balloon dilation. right: after balloon dilation using the Olbert Catheter System.

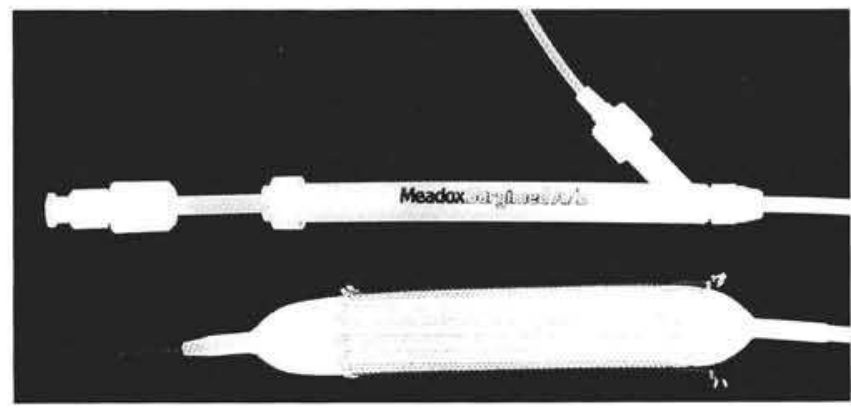

Figure 2: Olbert Catheter System (Meadox-Surgimed, Denmark) for esophageal dilation. The inflated balloon is seen inside an Ultraflex prosthesis, expanding it over its whole length with the 0.038-inch guide wire in the inner lumen. 
In ten patients suffering from malignant esophageal stenoses consistent balloon dilation of the implanted stent was carried out after implantation of an Ultraflex prosthesis that failed to expand adequately. In all cases, adequate complete expansion of the prosthesis, as well as secure fixation without prosthesis dislocation or esophageal dehiscence, was observed. In our view, regular balloon dilation using this new balloon catheter system is advisable when the guide wire is still in position in order to guarantee early functionality and secure fixation of the prosthesis in the tumor stenosis when an Ultraflex prosthesis initially fails to expand completely.

\section{N. Hoepffner, E. Foerster, W. Domschke}

Dept. of Medicine B, University of Münster, Münster, Germany

\section{References}

1. Cwikiel W, Stridbeck H, Tranberg KG, et al. Malignant esophageal strictures: treatment with a self-expanding nitinol stent. Radiology 1993; 187: 661-5.

2. Raijman I, Walden D, Kortan P, et al. Expandable esophageal stents: initial experience with a new nitinol stent. Gastrointest Endosc 1994; 40: 614-21.

Corresponding Author

N. Hoepffner, M. D.

Dept. of Medicine B

University of Münster

Albert-Schweitzer-Strasse 33

48129 Münster

Germany

Fax: $+49-251-837576$ 